\title{
Estudio de la comunicación en la evaluación de los diarios de prácticas que favorecen la argumentación
}

\section{Study of the communication in the evaluation of the diaries of practices that improve the argumentation}

\author{
Dr. Daniel Cebrián Robles \\ Universidad de Málaga, España \\ dcebrian@uma.es
}

\section{Dr. Rafael Pérez Galán}

Universidad de Málaga rpg@uma.es

\section{Dr. Manuel Cebrián de la Serna}

Universidad de Malaga mcebrian@uma.es

Cebrián Robles, D., Pérez Galán, R. \& Cebrián de la Serna, M. (2017). Estudio de la comunicación en la evaluación de los diarios de prácticas que favorecen la argumentación. Revista Prácticum, 2(1), 1-21. 


\section{Resumen}

Nada mejor para establecer un aprendizaje integrado entre los conocimientos de la facultad y las prácticas externas, que la metodología de los diarios donde los estudiantes reflexionan y argumentan sobre sus experiencias y aprendizajes. A pesar de ser estos diarios una práctica común en la mayoría de las instituciones, no siempre se realizan de forma dialógica y permanente con los tutores de la universidad, muchos menos entre los mismos estudiantes repartidos en diferentes centros, limitando así muchos de los beneficios que resultan de compartir las experiencias, más aún, el compartir las propias evaluaciones. El estudio con diseño cualitativo pretende analizar los resultados de las conversaciones entre tutor y estudiante en el momento de la evaluación, y el impacto de esta metodología para guiar y evaluar la reflexión y la argumentación sobre la práctica. Se analizaron 192 diarios estudiantes, y las 128 anotaciones que generaron desde análisis cualitativo con técnicas "Q-análisis". Al tiempo que, se presenta una metodología de rúbrica como el contenido de la misma para el seguimiento y evaluación de los diarios con criterios de argumentación. De las conclusiones más relevantes encontramos que se observa con claridad los cambios de mejora de la argumentación durante las catorce semanas.

\section{Abstract}

Nothing better to establish an integrated learning between faculty knowledge and external practices, than the methodology of the reflective daily where students reflect and argue about their experiences and learnings. Although these journals are a common practice in most institutions, they are not always done in a dialogical and permanent way with university tutors, much less among the same students distributed in different centers, thus limiting many of the benefits that result from sharing experiences, moreover, sharing the assessment themselves. The study with qualitative design aims to analyze the results of the conversations between tutor and student at the time of the assessment, and the impact of this methodology to guide and assessment the reflection and the argumentation about the practice. We analyzed 192 daily students, and the 128 annotations that generated from qualitative analysis with "Q-analysis" techniques. From the most relevant conclusions we find that the changes in argumentation improvement during the fourteen weeks are clearly observed. At the same time, a rubric methodology is presented as the content of the same for the monitoring and assessment of reflective daily with criteria of argumentation. 


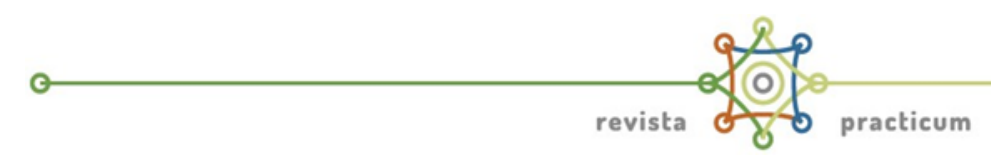

\section{Palabras claves}

Diario reflexivo; rúbrica digital; auto-evaluación, evaluación de pares y argumentación

\section{Key words}

Reflective daily; digital rubric; self-assessment, peer-assessment and argumentation

\section{Introducción}

Los diarios reflexivos pueden ser algo más que cuadernos donde los estudiantes recogen sus experiencias, y que valorados al final del curso con perspectivas de historias pasadas, una vez que ocurren, sin criterios explícitos antes o durante las prácticas. Sin duda, acotar la experiencia de las prácticas con criterios antes de que se produzcan, es como vaticinar qué va a ocurrir mañana. No obstante, sí podemos y debemos definir qué nivel de reflexiones y argumentos esperamos en los portafolios de los estudiantes, con independencia del tipo de experiencias que realicen, en el deseo de que pervivan estas buenas prácticas cuando sean docentes en activo (Zeichner \& Wray, 2001). De esta forma, podemos utilizar los diarios como una herramienta de aprendizaje, como dice Zabalza-Beraza (2013, pp.98), "un reposo reflexivo" -añadimos "en vivo y directo"- y como sigue señalando el autor que nos permite y "garantiza que no sólo está de prácticas, sino que vive y piensa la práctica que realiza". Entendemos que estas ventajas se consiguen mejor cuando se produce una comunicación dialógica con los docentes y demás estudiantes (Cebrian-de-la-Serna, 2011), y no lo dejemos "solos" reconstruyendo la narrativa de sus pensamientos y experiencias prácticas. Sin duda, en el centro tienen a los tutores que son los guías de estas experiencias, pero sabemos que a veces se encuentran con una tarea ingente en clase, y su tutoría está más orientada a cuestiones de solucionar los problemas de la práctica profesional, siendo el mayor valor sin duda del practicum, pero donde no siempre tienen oportunidad de seguir el pensamiento pedagógico y las reflexiones de su tutorando.

El hecho de no compartir las experiencias, no debatirlas y discutirlas, no reflexionar en "voz alta" y en el momento de producirse, dejándose para una revisión en la retrospectiva para la memoria final... impide, por un lado, recrear un pensamiento lógico y crítico más intenso, más directo y permanente con la realidad de los aprendizajes prácticos de 
forma contrastada con su tutor del centro. Y por otra, la mirada hacia atrás y el recuerdo a veces nos juega malas pasadas, y se apoya en ideas y preconcepciones. No teniendo más oportunidad para poder comprobar al día siguiente, que aquella idea que planteamos en el diario era errónea, acaso discutible... y que no mirábamos de la misma forma que lo hacía el tutor o los demás estudiantes. De este modo, el estudiante dispone de todo un conjunto de apoyos reflexivos, para enfrentarse de nuevo y cada día a los problemas de la práctica, aprendiendo a "mirar de otra forma" la realidad y las vivencias que le rodean, dando todo el sentido a las prácticas (Zabalza-Beraza, 2017).

Evidentemente, para tener esta inmediatez en la comunicación entre el estudiante y la universidad, necesitamos de una tecnología innovadora (Fernández Díaz \& Adelina Calvo, 2012; Bartolomé, Cantón, \& Ferrer, 2017), con procesos de recogida de información digital, de anotaciones y subidas de evidencias multimedia -imágenes, vídeos, sonidos...- (Cebrián-de-la-Serna, 2014; Gallego-Arrufat, \& Raposo-Rivas, 2014) y rúbricas digitales (Raposo-Rivas, Cebrián-de-la-Serna, \& Martínez-Figueira, 2013; Cebrián-de-la-Serna, \& Bergman, 2014) que acompañen y ofrezcan mayor información a los hechos e interpretaciones que dan los estudiantes... necesitamos de la tecnología que aumente nuestras experiencias recogidas en el portafolio digital desde una "comunidad de prácticas" (Klenowski, 2004), de intercambios de experiencias y valoraciones, de interpretaciones y argumentos que explican el porqué y el cómo de los hechos que interpretamos. Unido a la oportunidad de compartir soluciones y problemas que no se producen en su centro, y en cambio, sí en los demás centros y que son narradas por otros estudiantes.

Otra razón para acompañar las reflexiones prácticas son el nivel de profundidad que esperamos en los diarios. Esta reflexión analítica y deductiva necesita momentos donde los estudiantes escriben sus experiencias, las piensan y viven de nuevo con su narrativa interna, para después enfrentarse a la profundidad del debate y la defensa de nuestras experiencias y análisis (Zabalza-Beraza, 2004). Pues enfrentarse a la realidad como dialogar y debatir en profundidad, al tiempo que redactar por escrito lo aprendido, se produce con mayor nivel de calado y calidad en las reflexiones y argumentos cuando se hace colectivamente (Kim, 2013). Si a esto unimos criterios claros y objetivos que puedan servir de instrumentos para el debate, la reflexión y la argumentación, como para la propia evaluación de esta argumentación. Entonces tendríamos una herramienta valiosa para guiar la reflexión de las prácticas, y sobre todo, 


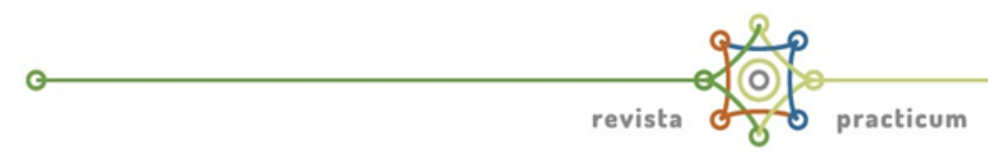

la evaluación de las mismas, con la seguridad de provocar una mayor calidad y profundidad argumental en las mismas.

\section{Autoevaluación y evaluación de pares sobre la argumentación en los diarios compartidos}

Si bien, hay una larga trayectoria científica sobre los diarios reflexivos (Klenowski, 2004; Marcolino, \& Reali, 2010; Bartolomé, Martínez-Figueira, \& Tellado-González, 2014) y en la argumentación en la formación inicial (Nussbaum, Sinatra, \& Poliquin, 2008; Kuhn, 2010; Cetin, Dogan, \& Kutluca, 2014). Sin embargo, no encontramos tantos trabajos que enfoquen los diarios reflexivos y la argumentación en las prácticas externas con rúbricas colaborativas (Özçinar, 2015) y con una contra argumentación y crítica reflexiva (Liu, \& Stapleton, 2014), además en línea (Baker, 2011). Mucho menos, con procedimientos y metodologías que permitan evaluar estos procesos reflexivos y argumentaciones. Algo similar sucede en los estudios de los procesos de regulación de los aprendizajes, la autoevaluación y evaluación de pares de forma colectiva y con rúbricas (Jenson, 2011; Cebrián-Robles, Serrano-Angulo \& Cebriánde-la-Serna, 2014). Pues estos nos permiten, no sólo ejercitar nuestro pensamiento e interpretación de las experiencias en el lugar donde realizamos las prácticas, sino compartir las mismas más allá del centro. Dando a conocer nuestras experiencias, problemas y sus soluciones, a otros estudiantes, multiplicando así las experiencias vividas, las propias y las ajenas; y que al evaluarlas entre pares en una comunidad virtual se sienten más próximas. Por tanto, consideramos cómo las nuevas líneas de investigación centradas en los procesos de aprendizaje de los alumnos deben estar orientados a través de herramientas como la erúbrica que potencia la autorregulación de los alumnos en su proceso de aprendizaje y le facilita compartir estas experiencias a través de grupos heterogéneos e interactivos en aras de una enseñanza de calidad para todos y a lo largo de toda la vida; también a través de entornos virtuales o de entornos semipresenciales o learning blended (Pérez, Cebrián \& Rueda, 2014).

La autoevaluación como la evaluación de pares por los propios estudiantes posee una extensa literatura (Martínez-Figueira, TelladoGonzález, \& Raposo-Rivas, 2013; Pérez-Torregrosa, Gallego-Arrufat, \& Gámiz-Sánchez, 2016), pero pocas veces se han producido estudios de estas variables cuando se realizan sobre experiencias en las prácticas externas. Experiencias reales y vividas "in situ" y en el momento, enfrentadas a problemas profesionales y en un espacio muy valorado y motivador siempre por los estudiantes (Tejada Fernández, 2005; Lawson, Çakmak, Gündüz, \& Busher, 2015). Por lo que, estamos en la creencia de 
que estos nuevos contextos incrementan en los estudiantes el impacto de las metodologías de la evaluación formativa, más bien diríamos que las multiplican; en cualquier caso, pocas veces se han estudiado estas metodologías en contextos de prácticas externas y de forma compartida. En suma, la evaluación es una oportunidad para aprender, es algo más que una calificación al final, es una comunicación durante el proceso de aprendizaje, donde estudiantes y docentes se intercambian valoraciones e interpretaciones sobre los aprendizajes. Esta comunicación no se ha estudiado en profundidad en contexto dialógico como los que se producen en los contextos de la evaluación de las prácticas, menos aún con tecnologías y criterios de argumentación como la rúbrica para los diarios.

\section{Métodología}

El estudio es parte de un proyecto más amplio de $I+D+i$ (1) donde se pretende conocer las posibilidades del uso de las tecnologías para la evaluación y seguimiento del prácticum. Para esto es necesario implementar y experimentar con pequeñas experiencias o grupos de estudiantes, que tras su evaluación puedan definir modelos de trabajo en grupos de estudiantes e instituciones más amplias. Por lo que, este trabajo es una muestra interesada de un proyecto donde hay otras instituciones -seis en total- que esperan recoger los resultados metodológicos de esta experiencia, como igualmente, éstas otras instituciones están realizando experiencias con otros grupos, contextos y modalidades diferentes en el uso igualmente con Corubric.com, plataforma donde se realizó la rúbrica de los diarios de prácticas.

El diseño de investigación es cualitativo con una intencionalidad descriptiva y orientada a la mejora (Buendía Eisman, Colás Bravo \& Hernández Pina, 1998, pp.4-6). En particular, y en esta experiencia, tenemos como objetivo experimentar la auto-evaluación y la evaluación entre pares mediado por la metodología y tecnología de la rúbrica, para conocer sus limitaciones y promover un aprendizaje más integral -entre el conocimiento práctico y académico- del estudiante a la hora de argumentar y reflexionar críticamente sobre sus experiencias de aprendizaje prácticos. Las técnicas de recogida y análisis de los datos fueron mediante un análisis cualitativo de categorías de análisis sobre las 128 anotaciones realizadas en cada una de las evidencias (Imagen $n^{\circ} 1 \mathrm{y}$ 2) producidas entre el docente y los estudiantes en el momento de la evaluación del diario de prácticas durante 14 semanas y desde los 192 diarios producidos. Estas anotaciones se exportaron a excel para su estudio. Los análisis de los datos se realizaron con categorías según la técnica de "Q-análisis" (Buendía Eisman, Colás Bravo \& Hernández Pina, 


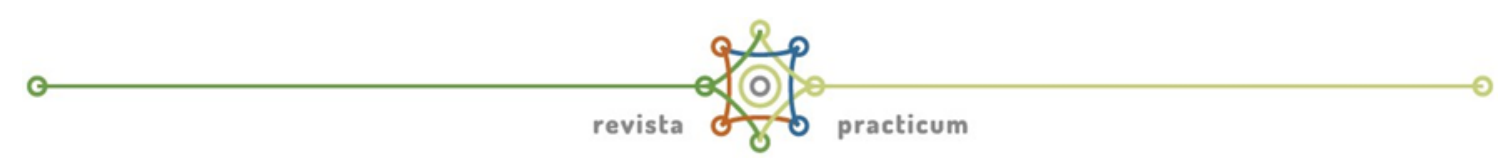

1998, pp.317-319) sobre los argumentos, evidencias y conclusiones de los estudiantes en los diarios de prácticas semanales, y que produjeron las anotaciones y conversaciones sobre su evaluación.

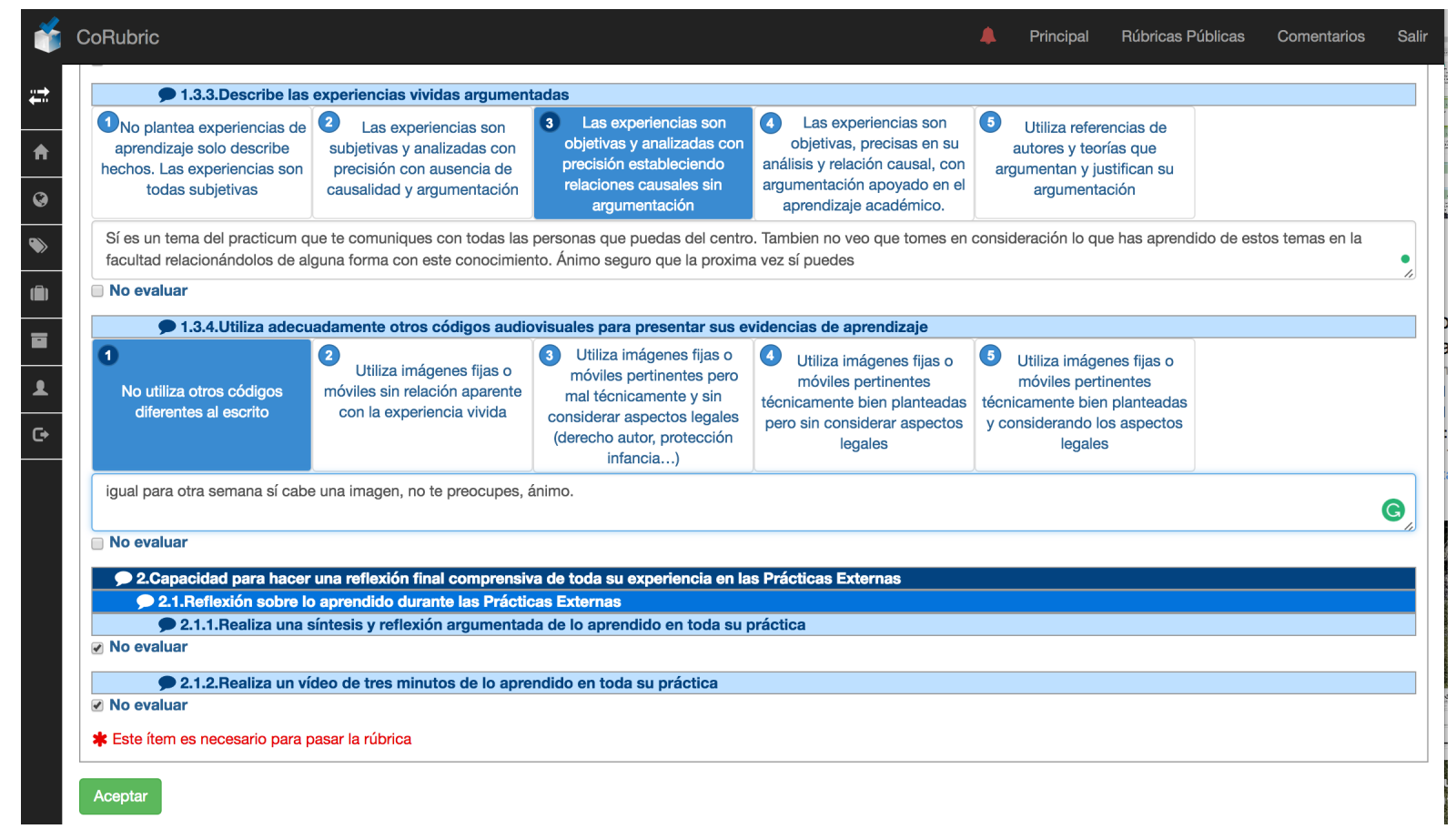

Ilustración 1. Observaciones realizadas por el docente y estudiante explicando cada calificación

En la imagen 1 podemos observar el texto que hay debajo de cada nivel de logro marcado, es una comunicación imprescindible para plantear una evaluación formativa, que da sentido y explicación cuando se presentan las evidencias, y explica la calificación junto con el alcance y posibilidad de mejora para el futuro. Por lo que, no se trata de calificar sino de establecer la calidad de la evidencia y explicar por qué este nivel de logro. 


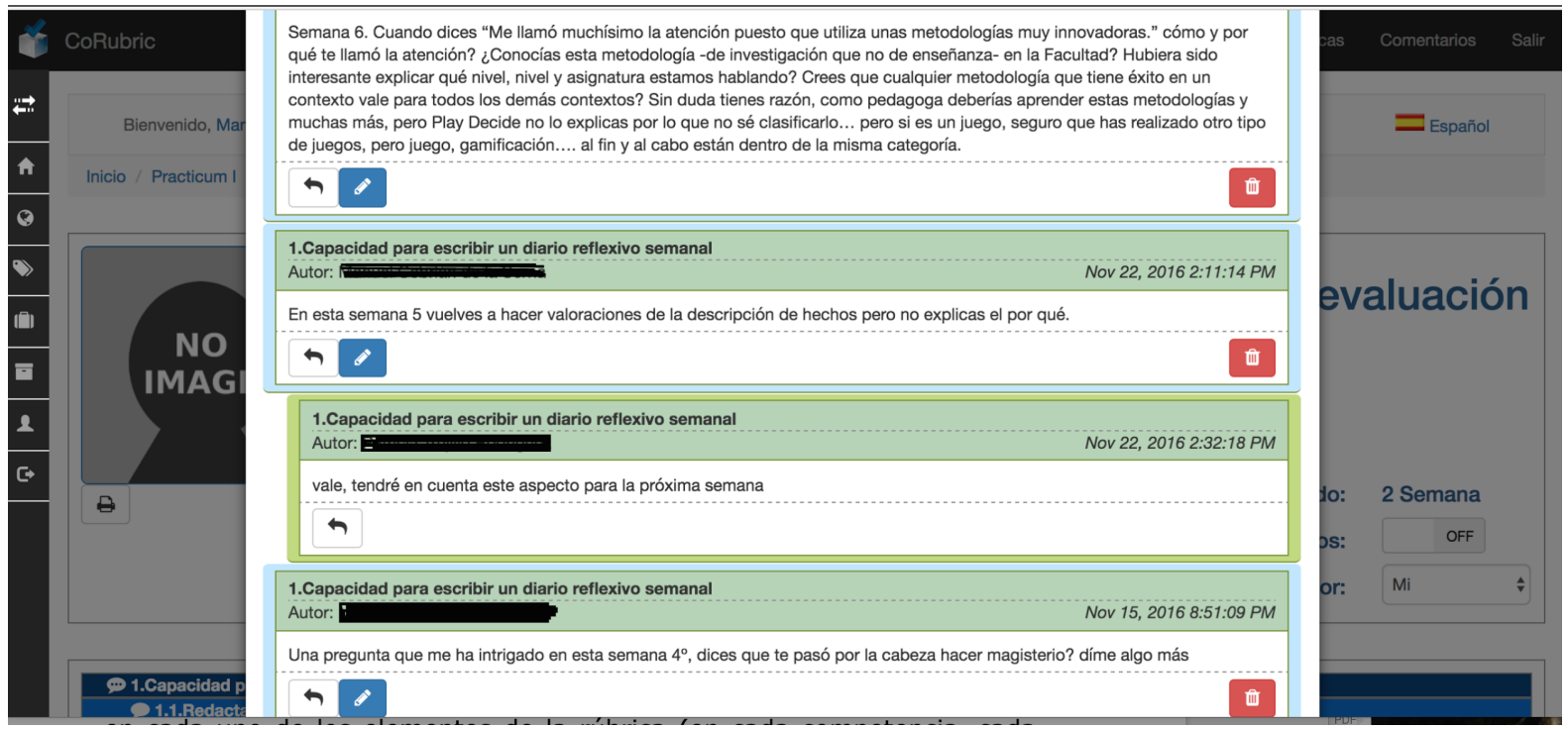

Ilustración 2. Anotaciones realizadas por los estudiantes y docentes en la conversación sobre la evaluación

Como podemos ver en la imagen 2, además del espacio para las observaciones que explican la calificación, existe un espacio para anotar en cada uno de los elementos de la rúbrica (en cada competencia, en cada indicador y en cada evidencia) una anotación que permite una comunicación evaluativa sobre otros aspectos de la comunicación humana, como la motivación al estudio, las consideraciones generales de los trabajos y conductas que observamos, los problemas en las prácticas...

\section{Descripción del contexto}

El grupo de estudiantes son 8 estudiantes de prácticum I (curso 1617 primer cuatrimestre) del grado de Pedagogía en tercer curso, Universidad de Málaga. Las prácticas se realizan en centros de secundaria, en empresas e instituciones varias (Museos, centros de la naturaleza, etc.) en todos los casos el estudiante debe presentar un portafolio al final o memoria de prácticas. Durante el curso se tutoriza de forma presencial y obligatoria (seminarios de formación al inicio, durante $y$ al final del cuatrimestre), durante la tutorización se realiza un seguimiento mediante un ePortafolios de evidencias multimedia (Monedero-Moya, Cebrián-Robles, \& Cebrián-de-la-Serna, 2015), donde se realiza una evaluación formativa y seguimiento semanal por el tutor académico -tutor de la universidad- mediante diferentes procedimientos, como son dos rúbricas analíticas que responden a todos los apartados que debe presentar el estudiante en su memoria final. Por un lado, en una 


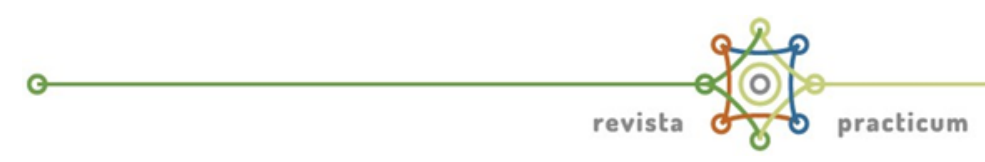

primera rúbrica realiza la evaluación y seguimiento de esta memoria con descripción del contexto y un plan de acción e intervención; por otro lado, una segunda está orientada exclusivamente al seguimiento y evaluación de los diarios, con un enfoque de favorecer la argumentación y reflexión crítica.

\section{Metodología de tutorización y evaluación de los diarios}

La segunda rúbrica o rúbrica de los diarios prácticos (ver cuadro no 1) lleva un seguimiento semanal de los diarios, que son en total 14 semanas o 14 diarios, que recoge uno o dos aspectos relevantes de su aprendizaje en esa semana en particular. La extensión máxima del diario semanal son 300 palabras, más orientado a establecer una conversación sobre un tema en profundidad que recoger las impresiones de toda una semana. De esta forma, se dispone también de más tiempo para responder y crear una conversación sobre un tema en particular.

Esta rúbrica se ha mejorado con el trabajo entre todos los docentes del proyecto, y en el que podemos observar cómo las evidencias pueden tener diferentes niveles de logro, circunstancia que hace más flexible que las rúbricas cuadradas a las que estamos acostumbrados. Esta flexibilidad también se produce en las diferentes ponderaciones o números cuantitativos que tienen estos niveles de logro, pudiendo comenzar unos por cero, otros por $1 \ldots$ como cambiar incluso los "escalones" entre estos niveles p.e. nivel 0 , nivel 2 , nivel 4 , nivel 8 de puntuación en cada nivel de logro. La ponderación es otra de las flexibilidades que posee esta rúbrica digital. Una evidencia puede tener un peso diferente con respecto a otra dentro de un indicador, y un indicador puede tener más o menos peso con respecto a los demás indicadores, igualmente los distintos pesos de las competencias.

\begin{tabular}{|c|c|c|c|}
\hline \multicolumn{4}{|c|}{ Competencia: 1.Capacidad para escribir un diario reflexivo semanal } \\
\hline \multicolumn{4}{|c|}{ Indicador: 1.1.Redacta sus experiencias con claridad } \\
\hline \multicolumn{4}{|c|}{ Evidencia: 1.1.1. Muestra una narrativa clara y vocabulario preciso } \\
\hline $\begin{array}{c}\text { Muestra una } \\
\text { narrativa clara } \\
\text { y vocabulario } \\
\text { preciso }\end{array}$ & $\begin{array}{c}\text { Se expresa } \\
\text { adecuadamente } \\
\text { y con claridad }\end{array}$ & $\begin{array}{c}\text { Trata } \\
\text { diversos temas } \\
\text { breves con } \\
\text { claridad y sin } \\
\text { relación } \\
\text { aparente }\end{array}$ & $\begin{array}{c}\text { Aborda } \\
\text { muchos temas } \\
\text { inacabados y } \\
\text { sin un lenguaje } \\
\text { apropiado }\end{array}$ \\
\hline \multicolumn{4}{|c|}{ (*) Evidencia: 1.1 .2 .Presenta un mensaje estructurado } \\
\hline $\begin{array}{l}\text { Ordena las } \\
\text { ideas y los }\end{array}$ & $\begin{array}{l}\text { Las ideas } \\
\text { están }\end{array}$ & $\begin{array}{l}\text { Define las } \\
\text { ideas pero no }\end{array}$ & $\begin{array}{l}\text { Presenta un } \\
\text { texto }\end{array}$ \\
\hline
\end{tabular}


Estudio de la comunicación en la evaluación de los diarios de prácticas que favorecen la argumentación

\begin{tabular}{|c|c|c|c|c|}
\hline & $\begin{array}{l}\text { hechos de } \\
\text { forma lógica y } \\
\text { estructurada }\end{array}$ & $\begin{array}{l}\text { relacionadas } \\
\text { entre ellas con } \\
\text { lógica }\end{array}$ & $\begin{array}{l}\text { están } \\
\text { relacionadas }\end{array}$ & $\begin{array}{l}\text { incoherente y } \\
\text { difícil de seguir }\end{array}$ \\
\hline \multicolumn{5}{|c|}{ Indicador: 1.2 .Utiliza correctamente el lenguaje escrito } \\
\hline \multicolumn{5}{|c|}{ Evidencia: 1.2 .1 .Ausencia faltas de ortografía } \\
\hline & & $\begin{array}{l}\text { No presenta } \\
\text { faltas de } \\
\text { ortografías }\end{array}$ & $\begin{array}{l}\text { No presenta } \\
\text { faltas de } \\
\text { ortografía } \\
\text { graves }\end{array}$ & $\begin{array}{l}\text { Presenta } \\
\text { importantes } \\
\text { faltas de } \\
\text { ortografía }\end{array}$ \\
\hline \multicolumn{5}{|c|}{ Evidencia: 1.2 .2 . Ausencia de incoherencias sintácticas } \\
\hline & & $\begin{array}{l}\text { Las frases } \\
\text { están bien } \\
\text { estructuradas } \\
\text { sintácticamente }\end{array}$ & $\begin{array}{l}\text { Las frases } \\
\text { son aún largas } \\
\text { pero bien } \\
\text { estructuradas }\end{array}$ & $\begin{array}{l}\text { Se observan } \\
\text { falta de } \\
\text { estructuración } \\
\text { sintáctica, con } \\
\text { frases largas }\end{array}$ \\
\hline \multicolumn{5}{|c|}{$\begin{array}{c}\text { Indicador: 1.3.Describe sus experiencias de aprendizaje con reflexión y precisión } \\
\text { argumental }\end{array}$} \\
\hline \multicolumn{5}{|c|}{$\begin{array}{l}\text { Evidencia: } 1.3 .1 . \text { Describe los procesos aprendidos más relevantes con } \\
\text { precisión }\end{array}$} \\
\hline $\begin{array}{l}\text { Describe los } \\
\text { procesos de } \\
\text { aprendizaje } \\
\text { vividos más } \\
\text { relevantes con } \\
\text { frases cortas y } \\
\text { precisas }\end{array}$ & $\begin{array}{l}\text { Describe los } \\
\text { procesos de } \\
\text { aprendizaje } \\
\text { vividos más } \\
\text { relevantes con } \\
\text { frases cortas y } \\
\text { precisas }\end{array}$ & $\begin{array}{l}\text { Describe los } \\
\text { hechos } \\
\text { relevantes con } \\
\text { frases cortas y } \\
\text { precisas }\end{array}$ & $\begin{array}{l}\text { Atiende los } \\
\text { aspectos más } \\
\text { relevantes de } \\
\text { los hechos pero } \\
\text { son frases } \\
\text { largas y } \\
\text { extensas }\end{array}$ & $\begin{array}{c}\text { Escribe } \\
\text { largas frases y } \\
\text { párrafos sin } \\
\text { centrarse en lo } \\
\text { esencial }\end{array}$ \\
\hline \multicolumn{5}{|c|}{ Evidencia: 1.3 .2 .Muestra una actitud de ética profesional } \\
\hline & $\begin{array}{c}\text { No muestra } \\
\text { frases o ideas } \\
\text { que atenten } \\
\text { contra la } \\
\text { dignidad de las } \\
\text { personas, las } \\
\text { instituciones, } \\
\text { los valores } \\
\text { democráticos y } \\
\text { la ética } \\
\text { profesional. }\end{array}$ & $\begin{array}{c}\text { Ha firmado } \\
\text { un contrato de } \\
\text { confidencialidad } \\
\text { con la } \\
\text { empresa. }\end{array}$ & $\begin{array}{l}\text { Utiliza un } \\
\text { lenguaje poco } \\
\text { profesional }\end{array}$ & $\begin{array}{c}\text { Utiliza un } \\
\text { lenguaje con } \\
\text { prejuicios que } \\
\text { atentan contra } \\
\text { la dignidad de } \\
\text { las personas, } \\
\text { las } \\
\text { instituciones, } \\
\text { los valores } \\
\text { democráticos y } \\
\text { la ética } \\
\text { profesional. }\end{array}$ \\
\hline
\end{tabular}

Evidencia: 1.3.3.Describe las experiencias vividas argumentadas

\begin{tabular}{|c|c|c|c|c|}
\hline $\begin{array}{l}\text { Utiliza } \\
\text { referencias de } \\
\text { autores y } \\
\text { teorías que } \\
\text { argumentan y } \\
\text { justifican su } \\
\text { argumentación }\end{array}$ & $\begin{array}{c}\text { Las } \\
\text { experiencias } \\
\text { son objetivas, } \\
\text { precisas en su } \\
\text { análisis y } \\
\text { relación causal, } \\
\text { con } \\
\text { argumentación } \\
\text { apoyado en el } \\
\text { aprendizaje } \\
\text { académico. }\end{array}$ & $\begin{array}{l}\text { Las } \\
\text { experiencias } \\
\text { son objetivas y } \\
\text { analizadas con } \\
\text { precisión } \\
\text { estableciendo } \\
\text { relaciones } \\
\text { causales sin } \\
\text { argumentación }\end{array}$ & $\begin{array}{c}\text { Las } \\
\text { experiencias } \\
\text { son subjetivas } \\
\text { y analizadas } \\
\text { con precisión } \\
\text { con ausencia } \\
\text { de causalidad y } \\
\text { argumentación }\end{array}$ & $\begin{array}{l}\text { No plantea } \\
\text { experiencias de } \\
\text { aprendizaje } \\
\text { solo describe } \\
\text { hechos. Las } \\
\text { experiencias } \\
\text { son todas } \\
\text { subjetivas }\end{array}$ \\
\hline \multicolumn{5}{|c|}{$\begin{array}{c}\text { Evidencia: } \begin{array}{c}\text { 1.3.4.Utiliza adecuadamente otros códigos audiovisuales para } \\
\text { presentar sus evidencias de aprendizaje }\end{array} \\
\end{array}$} \\
\hline Utiliza & Utiliza & Utiliza & Utiliza & No utiliza \\
\hline
\end{tabular}




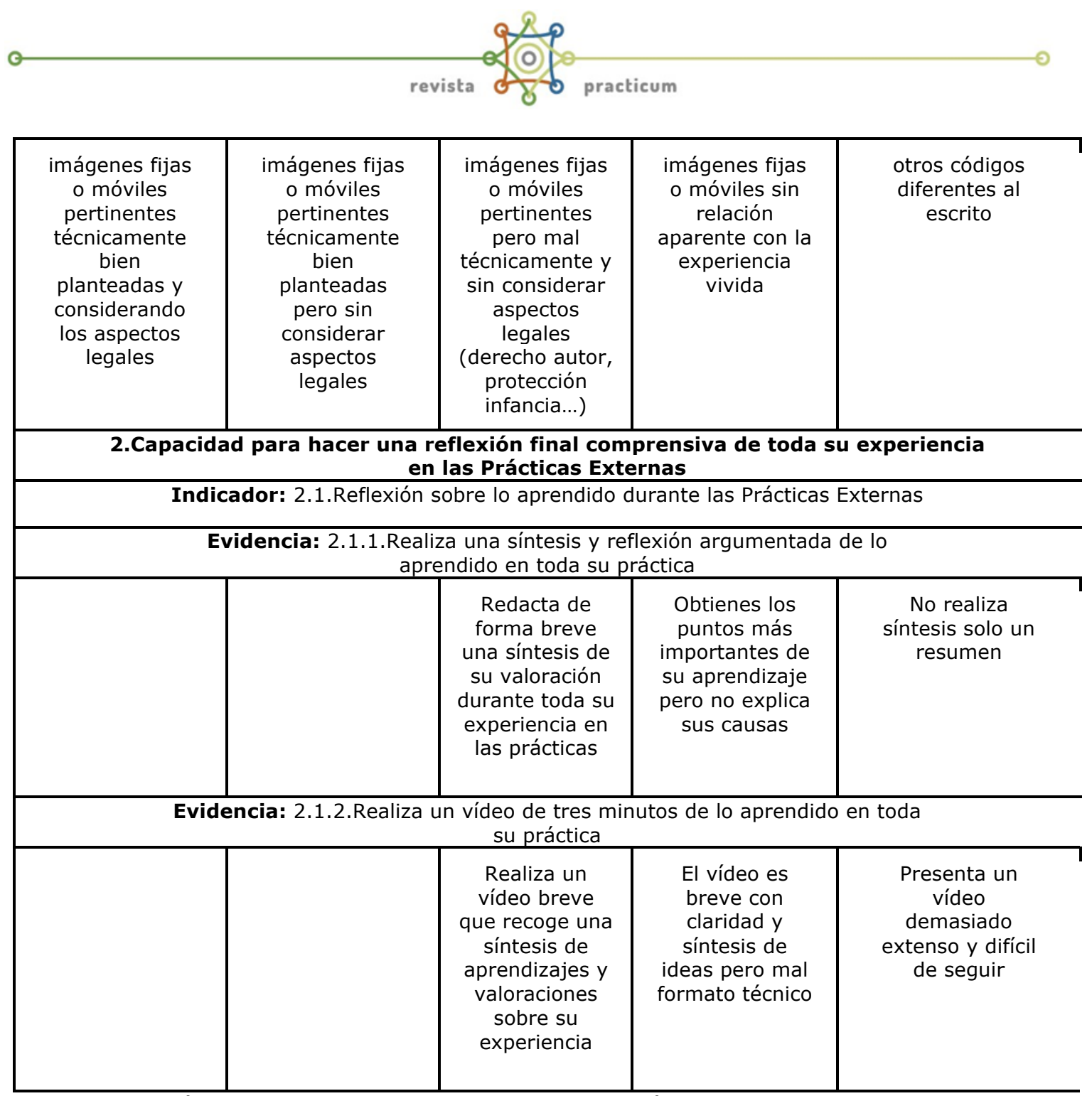

Tabla 1. Rúbrica de los diarios semanales (Elaboración propia) https://goo.gl/RIDM4M

(*) Este ítem es obligado para aprobar esta evidencia para aprobar toda la rúbrica

En este diario el estudiante reflexiona sobre las competencias exigidas al pedagogo como profesional en la institución/empresa en la que está desarrollando sus prácticas. Valora también estas competencias que considera necesarias para ejercer profesionalmente en ese puesto. Justifica su valoración y describe algún ejemplo extraído de la realidad de cada semana con sus prácticas de forma que avale sus afirmaciones. De alguna forma, estamos solicitando una argumentación o exposición de hechos, explicación de los elementos que lo constituyen y entrega de evidencias que demuestren lo que está exponiendo, o lo que es lo mismo: conclusión, justificación y evidencias. En el primer seminario presencial se explica esta rúbrica y se solicita una auto-evaluación de los criterios y niveles de logro reflejados en el texto que no se entienden, antes de realizar la primera evaluación. Realizando una confirmación de acuerdo, dado que esta rúbrica viene a ser una especie de "contrato" de trabajo. 
Cada semana los estudiantes suben su diario a la plataforma, se autoevalúan, evalúan a un compañero; y después de ese proceso, el docente realiza una evaluación y notas aclaratorias en el apartado de observaciones de CoRubric.com (ver imagen no 1 ) sobre la evaluación emitida; a la vez que, añade comentarios en el módulo de anotaciones que complementan esta evaluación (ver imagen no 2). El docente en todo momento y por semana tiene una aproximación conceptual de su trabajo, de cómo va evolucionando los estudiantes en conjunto sobre cada competencia, indicador y evidencia. Como se puede ver en la imagen no 3 se muestra una pantalla con la media que obtuvieron los estudiantes en la rúbrica del diario en la $3^{a}$ semana, que como es lógico, la media que es suspenso (ver en rojo el número arriba a la derecha de la imagen 42,48 o 4,2 de media), en general irá mejorando en lo sucesivo. También se puede llevar un seguimiento individual de un estudiante durante todas las semanas, y al final del proceso podemos exportar toda esta información en una hoja excel para su análisis. Si bien, visualmente también podemos observar esta situación cambiando la pestaña del número de semana (arriba a la derecha de la imagen no 3)

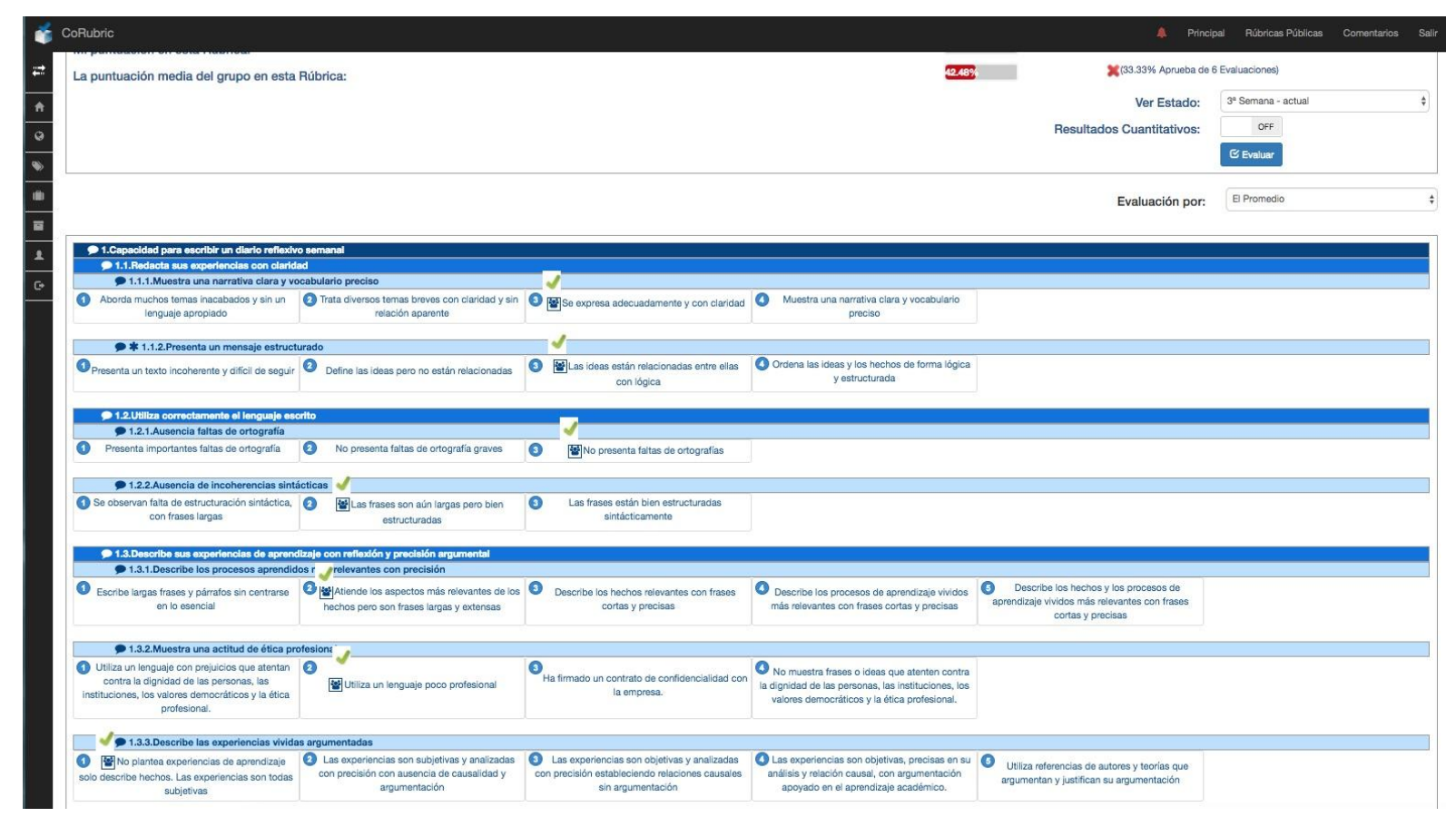

Ilustración 3. Visión de conjunto evolución global de los estudiantes semana 3

Como puede verse en esta imagen 3, el icono del grupo de estudiantes con el marcador en verde, señala la puntuación media y el texto cualitativo alcanzado por el grupo para una evidencia concreta en la semana 3. La evidencia 1.3.3. Describe las experiencias vividas 


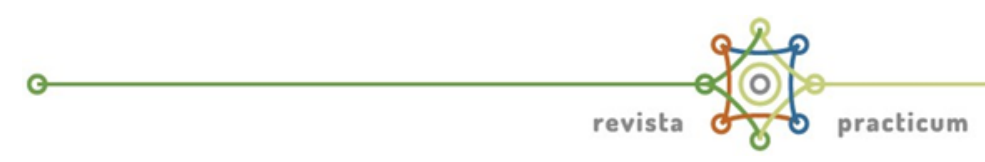

argumentadas, obtiene una puntuación media del grupo muy baja, y se indica cualitativamente con la frase: "No plantea experiencias de aprendizaje solo describe hechos. Las experiencias son todas subjetivas".

Es normal que en ocasiones el docente sugiera a veces $y$, por ejemplo, que haga partícipe a los demás estudiantes de esta experiencia, exponiendo al grupo un modelo interesante de atención a la familia, que aporte a los demás el recurso que encontró para evaluar a los estudiantes del aula, aquella herramienta útil para la productividad, etc. El docente en ocasiones sugiere que suban evidencias en formato de códigos multimedia para apoyar sus reflexiones (vídeos, sonidos, imágenes y texto...) y argumentaciones.

\section{Resultados}

\section{Análisis de los datos cualitativos.}

El número de anotaciones fueron 128 las analizadas. Contando el texto alcanzó 7.781 palabras. Las categorías de análisis fueron 9 que se obtuvieron desde la síntesis de las unidades mínimas o frases dentro del texto de las anotaciones: Redacción, Utilidad de la rúbrica, La crítica constructiva; Describir hechos, Síntesis, Inteligencia emocional, Códigos Multimedia y Aprendizaje práctico. A continuación, vamos a redactar algunas de las conclusiones que hemos observado en la lectura de estas conversaciones:

Redacción. Expresiones sobre la dificultad de redacción.

El inicio de redactar el diario es en palabras de los propios estudiantes un "calvario" y "desasosiego" para algunos. Las pautas que orientan cuando se utiliza la rúbrica suele ayudar a eliminar esta situación de partida, junto con ejemplos de otros años o anotaciones explicativas en los propios niveles de logro que permiten mostrar direcciones web, imágenes o cualquier material didáctico de apoyo. Son frases de los estudiantes las siguientes: "Estoy de acuerdo con la puntuación, pienso que este diario en general no lo he enfocado bien. Para mi era un trabajo complejo, puesto que nunca lo había realizado antes".

Utilidad de la rúbrica. Más motivado a escribir sin ningún "corsé" 
Suelen comenzar el diario sin mirar a la rúbrica, esto ocurre hasta la segunda o tercera semana. Suelen estar más animados a contar sus experiencias sin ningún "corsé" o criterio en general.

Les resulta difícil también comprender que las calificaciones son una orientación y no una calificación definitiva, circunstancia que va mejorando como las propias calificaciones y las argumentaciones a medida que evolucionan. Ambos aspectos -valoración, argumentación y calificación como orientación- se ven muy ayudados si el tutor establece una comunicación de confianza con frases como las siguientes: "Las calificaciones de la rúbrica... no te preocupes solo es para regular, orientar y tutorizar tu trabajo"; "Tengo que nuevamente felicitarte, porque veo que estás haciendo una labor importante e interesante para tu formación"; "Tengo que felicitarte por tu trabajo y porque dices estás "nutriéndote" de aprendizajes... pues explica esta nutrición con más detalles"; "Ánimo, vas muy bien, no tengas en cuenta tanto las calificaciones que te salgan sino el porqué de tu reflexión y argumentación... en este caso sólo te quedaste en la evidencia, en los hechos, poco en lo que aprendiste, de las conclusiones que obtienes de esta experiencia, de las referencias leídas en la facultad, etc"; "En cuanto a la experiencia, enhorabuena, la primera vez que uno tiene que enfrentarse a un público es muy importante. No me hablas de cómo te preparaste, de qué estrategia diseñaste previamente, de qué recursos o ejemplos te valiste de tu experiencia en la universidad, etc."

A veces hemos tenido que subrayar la realidad del mundo laboral cuando se encontraban repetidamente viendo que la práctica no era todo un "divertimento", con frases del tutor académico como: "Evidentemente estas tareas se llaman "trabajo" y las cobran porque es una tarea profesional, ocupa y suele ser "trabajoso". Estás en el mundo profesional, es normal"; "Hola XXXX, la verdad es que escribir los diarios está siendo una difícil tarea puesto que siempre me tienen haciendo lo mismo y ya no se que más puedo poner de un aprendizaje inexistente". evaluación.

La crítica constructiva. Dificultad para diferenciar matices en la

Todos somos emocionalmente sensibles cuando se realiza una evaluación, incluso cuando hay una crítica profesional de los actos de uno o de los demás. En el mundo profesional el trabajo en equipo es muy importante, y se necesita competencia para poder aportar ideas y mejoras al grupo, como soportar las críticas sobre uno desde los demás compañeros. Son conversaciones como la siguiente de una estudiante que tuvo un problema al exponer sus opiniones de mejora sin medir la 


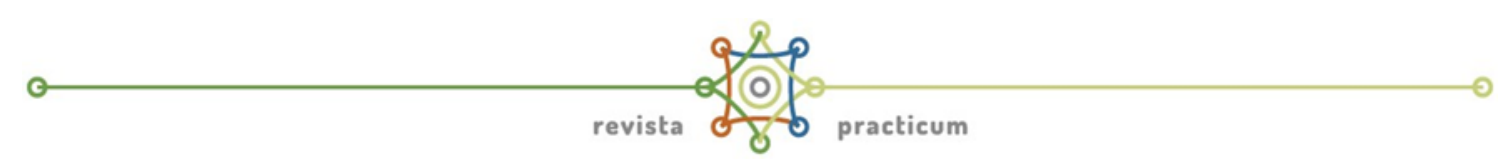

situación de cómo y dónde se exponía: "Hola, felicidades porque presentas una innovación o mejora de procesos y además lo demuestras con evidencias. No pensé mal en cuanto a lo que dices de crítica, yo creo que es bueno la crítica, otra cosa es poner en evidencia -se refiere al hecho de poner en evidencia al jefe-. El problema es si estamos preparados o existe un ambiente para la crítica constructiva".

Describir hechos. Descripción de hechos sin interpretar hasta llegar a la argumentación.

Por lo general y desde el principio, suelen describir hechos uno tras otro sin valoración, sin impresiones, explicaciones del porqué de lo que ven, observan y escuchan... pareciera que posee mayor valor: describir cuanto más mejor, sin comprometerse con ninguna explicación. $Y$ es un "caballo de batalla" en casi la mitad del cuatrimestre. Difícil les resulta a todos, como puede observarse en la imagen 3, donde en la semana 3 se ilustra con un ejemplo de esta situación: la evidencia 1.3.3. va mejorando a medida que toman más confianza con el tutor y comprenden que tienen catorce oportunidades más para redactar bien su diario. Cuando llegan al final y revisan todo lo escrito ellos mismo observan esta evolución. Lo que sirve de ayuda al docente para tener una visión de conjunto del grupo. Encontramos frases en las primeras semanas, como: "...durante los días 18 y 19 me pusieron a trabajar pasando los contenidos de una unidad didáctica a Exelearning utilizando los criterios pedagógicos... En estos días, nos dieron la oportunidad de asistir a una reunión con XXX sobre el uso de Articulate Storyline... Al finalizar la semana, XXX me pidió que escribiese un post...". En síntesis, se comienza con descripción de hechos que hay que mejorar en la redacción, para comenzar a partir de la $3^{a}$ semana un proceso de valoraciones, opiniones, etc. que hay que fundamentar con más información y documentación. En las últimas semanas se alcanza una mejoras importantes en la descripción, con reflexiones más o menos profundas, y en donde las evidencias comienzan a mostrar mayor utilidad, pues en un inicio no suele tener relación con la argumentación, menos aún tomar iniciativas para volver a comprobar sus hipótesis. Como este estudiante en la semana 9 donde después de escuchar comentarios de los docentes hacia el fracaso de ciertos estudiantes, comenta: "En los siguientes días trataré de entrar en las clases de estos profesores para ver como trabajan, ya que en mi opinión, una buena metodología que atiende a todos los alumnos y los considera a todos importantes es la clave para el éxito. Y puede ser que ahí radique la causa del fracaso escolar de estos alumnos en esas asignaturas." 
Síntesis. Dificultad para sintetizar una idea con relativa profundidad.

Realizar una síntesis es difícil para los estudiantes, igual que redactar frases breves y atenerse a las 300 palabras, como también centrarse en un tema y analizarlo con cierta profundidad. Para todo esto la orientación y conversación con el tutor es fundamental, buscando este análisis y argumento con preguntas directas, y centrándose en un tema, promoviendo que revise lo sucedido para la semana próxima y observe de nuevo aquel hecho descrito pero con otra "mirada". Ejemplo como los siguientes, cuando dice el tutor: "No señalas qué competencias no aprendistes cuando dices "A nivel práctico no estaba preparada, en muchas ocasiones echaba en falta capacidades y herramientas que quizás tendríamos que haber trabajado con anterioridad en la facultad" ¿cuáles dice el tutor-? y cuando dices "no presentan nuevos retos ni enseñan a adoptar la postura autónoma, proactiva y real que creo que precisa un buen pedagogo" pregunta el tutor "¿cómo crees que se podría conseguir esto de la mejor forma?"

\section{Inteligencia emocional. ¿La gran olvidada?}

A veces la comunicación permite un apoyo más emocional, pues como todo trabajo profesional, en numerosas ocasiones se encuentran "perdidos" y en momentos "pocos excitantes", en palabra de los estudiantes, suelen tener expectativas altas y a veces equivocadas de lo que es el mundo profesional. Existe una gran diferencia entre los centros escolares -institutos para los pedagogos, en general- frente a los museos y especialmente de las empresas de formación. En esta últimas, suelen poner a prueba la gestión emocional de los estudiantes, sobre todo cuando se enfrentan con estructuras bien marcadas y ambientes muy estresantes. Aquí la tutoría puede en ocasiones no ser suficiente para que los estudiantes abandonen y soliciten otro centro. Mucho más cuando entre ellos se comunican en la plataforma y encuentran más divertido y excitante el trabajo de los demás en centros de secundaria. Son frases de los estudiantes como "no estoy nada contento con mis prácticas y pienso que debería ser algo positivo y placentero para mí". De los tutores: "Lo importante es aprender de los errores, analiza qué sucedió para mejorarlo en la próxima ocasión. Estamos en formación y evaluación continua. Venga, ese es la actitud, proactiva, ánimo".

Códigos Multimedia. Competencia multimedia.

En la evidencia 1.3.4., "Utiliza adecuadamente otros códigos audiovisuales para presentar sus evidencias de aprendizaje", suelen tener dificultades en un principio, y requiere de formación e información previa 


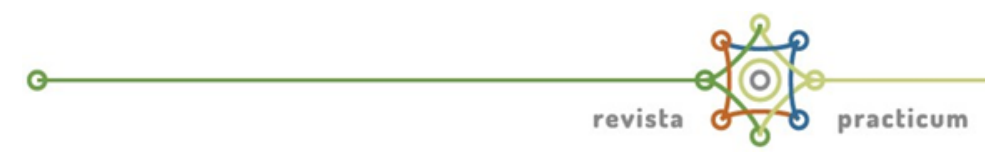

de cómo, cuándo, qué... evidencias presentar. Pero al final, el adjuntar material audiovisual ha permitido una oportunidad única para debatir sobre el derecho a la intimidad y preservación de la identidad de la infancia, la seguridad de las redes y la preservación de los derechos de autor y la confidencialidad firmada en el contrato con las empresas, como puede ser en cuanto a bases de datos de clientes, estrategias de los proyectos, etc. Es una experiencia y temática interesante que no todos la viven directamente pero que al menos se debate y se conoce en las evaluaciones de pares. Podemos no requerir esta evidencia en este tipo de formato, "escondiéndola bajo la alfombra", pero el problema y la necesidad de formación seguirán ahí en los estudiantes.

Aprendizaje práctico. Dificultad de relacionar el aprendizaje práctico con el conocimiento en la universidad.

Otras de las cuestiones que más difícil resulta presentar como evidencias consiste en establecer un vínculo entre los conocimientos que disponen o han sido adquiridos en la universidad, con el análisis de las experiencias que se viven en los centros de prácticas. Esto pudiera ser así, porque muchos de los conocimientos de la universidad no se han planteado con un sentido más allá de superar una prueba o examen. También se corre el peligro de que las prácticas sean "un estar", y no un pensar y reflexionar sobre lo aprendido en ellas. Como igualmente la práctica diaria es muy absorbente y dados los estudiantes más a hacer que a pensar, pudiera ser el motivo por el cual ni siquiera revisan la rúbrica en las dos primeras semanas. Basta con estar y trabajar, como también puede suceder en la universidad, pensar que basta con estar sentado en la silla de clase. Frases como esta suelen ser frecuentes, cuando dice el tutor "... en este caso solo te quedastes en la evidencia, en los hechos, poco en lo que aprendistes, de las conclusiones que obtienes de esta experiencia, de las referencias leídas en la facultad, etc."

\section{Conclusiones}

Realizando una síntesis podemos estar más convencidos de que la metodología de autoevaluación y evaluación de pares, junto con las conversaciones y comunicaciones generadas gracias a la rúbrica, han mostrando su eficacia para la orientación y guía de las reflexiones, como se comprueba también en otros trabajos (Toom, Husu \& Patrikainen, 2015), con los criterios y recogida de evidencias para un aprendizaje auténtico (Zeichner, \& Wray, 2001, pp620). Igualmente, se ha generado una tutoría más allá de la argumentación y crítica de la práctica, 
atendiendo a los aspectos más emocionales y otros aspectos que surgen de aprendizajes muy profesionales, únicos y singulares de la práctica. Antes de que los estudiantes comiencen las prácticas, resulta importante preparar para analizar y conocer sobre las expectativas que puedan tener. Para esto, en los seminarios previos es muy útil analizar los vídeos y algunos textos de los estudiantes de años anteriores. Salvo excepciones, por lo general, los estudiantes muestran muy buena disposición a aprender en las prácticas. Gracias a la tecnología de la rúbrica digital se ha permitido desarrollar una evaluación formativa en la distancia, una verdadera comunidad de práctica y una evaluación comunicativa. No dejándolos "aislados" a su suerte en la redacción de la narrativa personal que implica aprender en un contexto profesional, sin tutoría o mediante metodologías menos instantáneas $\mathrm{y}$, en todo momento, desde la universidad. Con ello, podemos observar un cambio cualitativo en la argumentación que presentan sin duda dificultadas como en otros trabajos (Nussbaum, Sinatra, \& Poliquin, 2008; Iordanou, \& Constantinou, 2014), donde argumentar es más difícil que buscar pruebas. Siendo optimistas en cualquier caso, y por dos razones, una porque son estudiantes del Practicum I y disponen aún de otro cuatrimestre para mejorar los resultados; dos porque hemos tenido éxito en otras materias en el impacto de la rúbrica (Cebrián-de-la Serna, Serrano Angulo, \& Ruiz Torres, 2014), solo que en otro contexto y asignaturas más presenciales.

La innovación educativa tiene algo de aventura por lo que significa adentrarse en territorios "pocos transitados" (Barrett, 2009) y buscar los límites de las posibilidades para el cambio, como asumir las tensiones y los retos que solicita a los actores de la innovación (docentes, estudiantes, familias...), para transformar los obsoletos o inadecuados pensamientos y conductas en buenas prácticas educativas. Este caminar se hace más fácil cuando estamos y nos sentimos acompañados, con una conversación en comunidades de aprendizaje y de prácticas, con criterios e indicadores de buenas prácticas como las que hemos expuesto en el presente trabajo.

\section{Nota}

1. Proyecto del Plan Nacional I+D+i 2014-17. Estudio del impacto de las erúbricas federadas en la evaluación de las competencias en el practicum. Plan Nacional de I+D+i de Excelencia, no EDU201341974P. web: http://goo.gl/u07aNs 


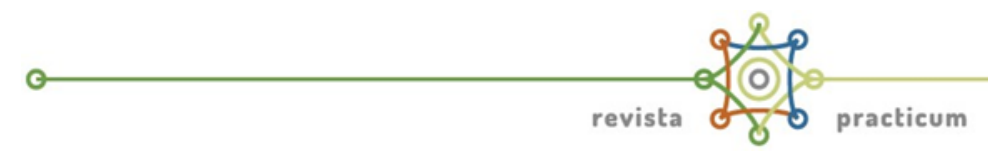

\section{Referencias}

Bartolomé, A., Cantón, I. C. \& Ferrer, J. M. M. (2017). Una revisión a los Practicum de Educación desde las tecnologías. Revista Prácticum, 1(1).

Bartolomé, A., Martínez-Figueira, E., \& Tellado-González, F. (2014). La evaluación del aprendizaje en red mediante blogs y rúbricas: ¿complementos o suplementos? REDU.Revista De Docencia Universitaria, 12(1), 159-176.

Barreto, H. (2009). Conferencia inaugural. Jornadas Internacionales "Docencia, investigación e innovación en la universidad. Trabajar con ePortfolios". Universidad de Santiago de Compostela (España) 23-24 nov.

Baker, D. L. (2011). Designing and orchestrating online discussions. Journal of Online Learning and Teaching, 7(3), 401-411.

Buendía Eisman, L., Colás Bravo, P., \& Hernández Pina, F. (1998). Métodos de investigación en psicopedagogía. Madrid: McGraw-Hill.

Cebrián-de-la-Serna, M.; Serrano-Angulo, J. \& Ruiz-Torres, M. (2014). Las eRúbricas en la evaluación cooperativa del aprendizaje en la Universidad. Comunicar, 43.153-161.https://doi.org/10.3916/C43-201415.

Cebrián-de-la-Serna, M. \& Bergman, M. (2014). Evaluación formativa con e-rúbrica: aproximación al estado del arte. REDU. Revista de docencia universitaria. V 12 (1) 15-22. https://doi.org/10.4995/redu.2014.6427

Cebrián Robles, D., Serrano Angulo, J., \& Cebrián de la Serna, M. (2014). Federated eRubric service to facilitate self-regulated learning in the european university model. European Educational Research Journal, 13(5), 575-583. doi:10.2304/eerj.2014.13.5.575

Cebrián de la Serna, M. (2014). El ePortafolio de evidencias y la evaluación formativa con eRúbricas. Revista EmRede, 1(1), 7-20.

Cebrián de la Serna, M. (2011). Supervisión con ePortafolios y su impacto en las reflexiones de los estudiantes en el Practicum. Estudio de Caso. Revista de Educación, no 354, Enero. pp.183-208.

Cetin, P. S., Dogan, N., \& Kutluca, A. Y. (2014). The Quality of Preservice Science Teachers' Argumentation: Influence of Content Knowledge. Journal of Science Teacher Education, 25(3), 309-331.

Fernández Díaz, E., \& Adelina Calvo, s. (2012). La formación permanente del profesorado en el uso innovador de las TIC. Una investigación-acción en infantil y primaria. Profesorado. Revista de currículum y formación de profesorado, 16(2), 355-370. 
Gallego-Arrufat, M.J., \& Raposo-Rivas, M. (2014). Compromiso del estudiante y percepción del proceso evaluador basado en rúbricas. REDU. Revista De Docencia Universitaria, 12(1), 197-215.

Iordanou, K., \& Constantinou, C. P. (2014). Developing pre-service teachers' evidence-based argumentation skills on socio-scientific issues. Learning and Instruction, 34, 42-57.

http://doi.org/10.1016/j.learninstruc.2014.07.004

Jenson, J. D. (2011). Promoting self-regulation and critical reflection through writing students' use of electronic portfolio. International Journal of ePortfolio, 1(1), 49-60.

Kim, S. H. (2013). The Effects of Reflection Rubric on Critical Thinking and Collaboration Argumentation in CSCA environment. Journal of the Korea Academia-Industrial cooperation Society, 14(11), 5559-5569.

Klenowski, V. (2004). Desarrollo del portafolios para el aprendizaje y la evaluación: Procesos y principios. Madrid: Narcea Ediciones.

Kuhn, D. (2010). Teaching and learning science as argument. Science Education, 94(5), 810-824. doi:10.1002/sce.20395

Lawson, T., Çakmak, M., Gündüz, M., \& Busher, H. (2015). Research on teaching practicum - a systematic review. European Journal of Teacher Education, 38(3), 392-407. doi:10.1080/02619768.2014.994060

Liu, F., \& Stapleton, P. (2014). Counter argumentation and the cultivation of critical thinking in argumentative writing: Investigating washback from a high-stakes test. System, 45, 117-128.

Marcolino, T. Q., \& Reali, A. M. (2010). Análisis de los feedbacks de diarios reflexivos a lo largo de un proceso de mentoría en grupo. Revista Iberoamericana de Educación, (52) 6. https://goo.gl/JvXGh6

Martínez-Figueira, E., Tellado-González, F., \& Raposo-Rivas, M. (2013). La rúbrica como instrumento para la autoevaluación: Un estudio piloto. REDU. Revista De Docencia Universitaria, 11(2), 373-390

Monedero-Moya, JJ.; Cebrián-Robles, D. y Cebrián-de-la-Serna, M. (2015). Documentando el eportafolios federado con evidencias multimedia, anotaciones de video y erúbricas. XIII Symposium Internacional sobre el Practicum y las Prácticas Externas. 29Jun-1Jul 2015. Poio. Pontevedra. https://goo.gl/UOiyt8

Nussbaum, E. M., Sinatra, G. M., \& Poliquin, A. (2008). Role of epistemic beliefs and scientific argumentation in science learning. International 


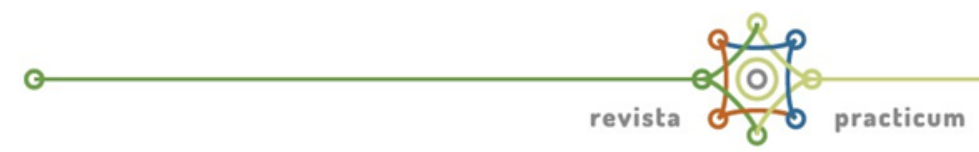

Journal of Science Education, 30(15), 1977-1999. doi:10.1080/09500690701545919

Özçinar, H. (2015). Scaffolding computer-mediated discussion to enhance moral reasoning and argumentation quality in pre-service teachers. Journal of Moral Education, 44(2), 232-20. doi: $10.1080 / 03057240.2015 .1043875$

Pérez Galán, R., Cebrián Robles, D. \& Rueda Galiano, A.B. (2014). Evaluación de pares y autoevaluación con erúbricas: caso de estudio en el grado de Educación Primaria. REDU - Revista de Docencia Universitaria, 12 (4), 337-456. Recuperado de http://www.red-u.net

Pérez-Torregrosa, A.B., Gallego-Arrufat, M.J., \& Gámiz-Sánchez, V.M (2016). Self Assessment with Electronic Rubrics of Undergraduates in the Practicum in Spain and Greece. Paper presented at the ECER 2016, Leading Education: The Distinct Contributions of Educational Research and Researchers. European Educational Research Association, EERA.

Raposo-Rivas, M.,Cebrián-de-la-Serna, M.,\& Martínez-Figueira, S. (2013). The electronic rubric to value skills on ICT subjects. European Educational Research Journal. (13) 5, 584-594. https://doi.org/10.2304/eerj.2014.13.5.584

Tejada Fernández, J. (2005). El trabajo por competencias en el prácticum: Cómo organizarlo y cómo evaluarlo. Revista Electrónica De Investigación Educativa, 7(2), Recuperado de https://goo.gl/45eRbi

Toom, A., Husu, J., \& Patrikainen, S. (2015). Student teachers' patterns of reflection in the context of teaching practice. European Journal of Teacher Education, 38(3), 320-340. doi:10.1080/02619768.2014.943731

Zabalza-Beraza, M. (2017). El Practicum y las prácticas externas en la formación universitaria. Revista Prácticum, 1(1). https://goo.gl/4I7h55

Zabalza-Beraza, M. A. (2013). El practicum y las prácticas en empresas en la formación universitaria. Madrid: Narcea Ediciones.

Zabalza-Beraza, M. A. (2004). Diarios de clase: Un instrumento de investigación y desarrollo profesional. Madrid: Narcea Ediciones.

Zeichner, K., \& Wray, S. (2001). The teaching portfolio in US teacher education programs: What we know and what we need to know. Teaching and Teacher Education, 17(5), 613-621. doi:10.1016/S0742051X(01)00017-8 